\title{
Design and Experimental Study of Rotary Type High-Speed Shearer for Metal Bars
}

\author{
Renfeng Zhao ${ }^{1}$, Jingyu Zhou ${ }^{1}$, Yan $\mathrm{Li}^{1}$, Shuqin Fan ${ }^{2}$, Jingxiang $\mathrm{Li}^{2}$, Xudong Xiao ${ }^{1}$ and Zhi Yang ${ }^{1}$ \\ ${ }^{1} X i$ 'an University of Technology, College of mechanical and precision instrument engineering, xi'an, 710048, China \\ ${ }^{2} X i ' a n$ Jiaotong University, School of Mechanical Engineering, xi'an, 710049, China
}

\begin{abstract}
For the precision shear of bars, the kind of rotating high-speed precision cutting method has been given. The structure design of rotary precision shearing machine and the structure design of feeding structure are completed. The method of numerical simulation is used to optimize the cutting tool, and then the experiment platform is completed. Rotary impact and the stress concentration effect of the surface of the bar are used in the paper. The experiment is carried out for the $\Phi 8 \mathrm{~mm}$ diameter stainless steel bar stock by changing the speed of the cutting tool through the converter and controlling rod feeding efficiency. The experimental results show that the method is feasible to meet the requirement of various material of the small diameter rod in the industry.
\end{abstract}

Keywords: Bar; High-speed Shearing; Stress Concentration; Cross-section Quality

\section{Introduction}

Metal bars are widely used in the industrial production. The traditional blanking methods which are sawing, turning and punching and shearing blanking[1-3]can not meet the new requirements of industrial production. Therefore, it is of great significance to study the new blanking method. But, the sawing and cutting blanking are not only a waste of raw materials, but also the efficiency is low. The quality of impact shear blanking method is poorer. High energy consumption blanking process, such as laser cutting can only cut the plate and pipe with medium and small thickness, blanking efficiency is low, and equipment cost is high. The high pressure water jet cutting equipment is expensive, the abrasive price is higher and lower precision than laser cutting. The method of gas cutting has an effect on the composition and tissue on both sides of the incision, and can cause deformation of the workpiece. The overall efficiency is low when the mass is cut[4]. Therefore, the traditional blanking method and high energy consumption blanking process already can not meet the requirement of the new type of industrial production[5-6]. It is of great significance to study the new blanking method. So, we need to carry out careful design and research on the blanking equipment. This paper discusses the rotary shear blanking machine, high-speed shear principle and the rotary cutting mechanism are used[7,8], flywheel every rotation can work twice for blanking, greatly improving the efficiency of blanking.

\section{The working principle of rotary high speed blanking machine}

The schematic diagram of the high speed rotary shear blanking machine is shown in Fig.1.

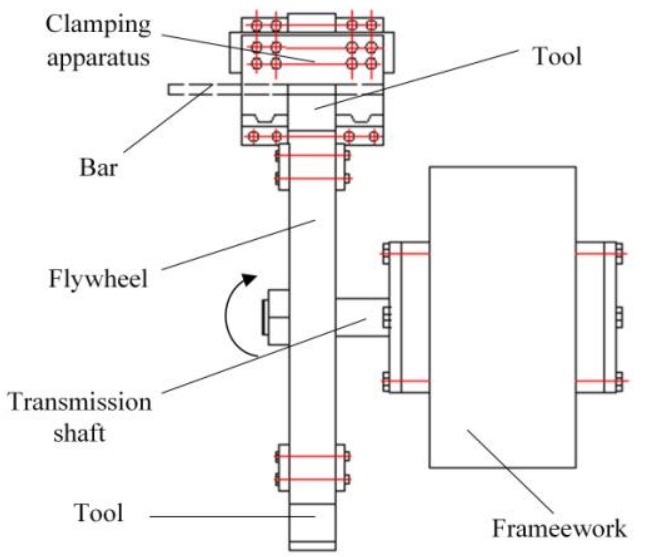

Figure 1 Rotary Shearing Principle Diagram

The rotary type high-speed shearing blanking machine includes the blanking mechanism, the clamping mechanism and the feeding mechanism, and the three parts together complete the cutting blanking of the metal bar. The blanking mechanism and the clamping mechanism directly affect the section quality of the metal bar, which is the core part of the rotary shear blanking machine. The feeding mechanism is the auxiliary mechanism. The main design of the blanking part is based on the diameter of the bar which needs to be cut.

In the cutting process, the bar is fixed with fixture, and the tool can be used for rotary shear. The bar is cut by relative movement of cutting tool and clamp. Gao[8] divided the shear process into elastic stage, plastic 
deformation stage, crack generation and fracture stages, we also divided into elastic stage, stage of plastic deformation, crack, and separation stage, as shown in Fig.2.

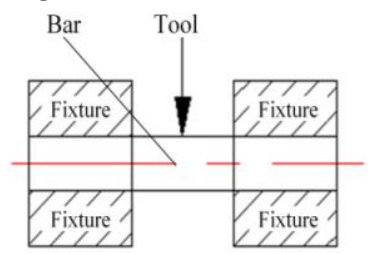

(a)

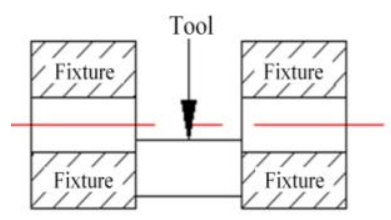

(c)

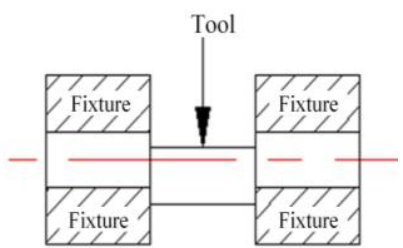

(b)

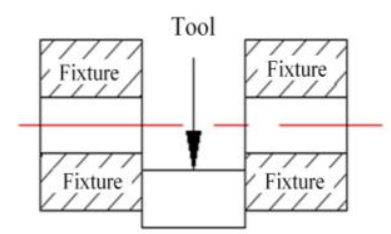

(d) (a) Elastic Stage (b) Plastic Deformation Stage (c) Crack Formation (d) Separated Stage

Figure 2 High Speed Shearing Process Diagram

The basic working principle of the feeding machine is: the feeding mechanism adopts wheel transfer power, and the flywheel can complete the storage realization. The motor is driven firstly, the wheel transfer motion to complete the energy storage of the flywheel. The flywheel can achieve the function of the blanking by releasing energy. The clamping device is connected with the blanking platform by bolted connection. Rotary shear blanking machine need to cut the bar that with the same length and different diameter, that is why the mould clamping structure uses double V-type blocks. In order to replace the die easily, the traditional bolt connection is replaced by the hydraulic cylinder, and the stability and speed of clamping are guaranteed. The rod feeding mechanism is not very important in the whole shear system, but the accurate feeding is also an indispensable part of successful blanking.

\section{Structure design of blanking system}

\subsection{The cutting tool structure}

The structure design of the cutting tool is shown in Fig.3.

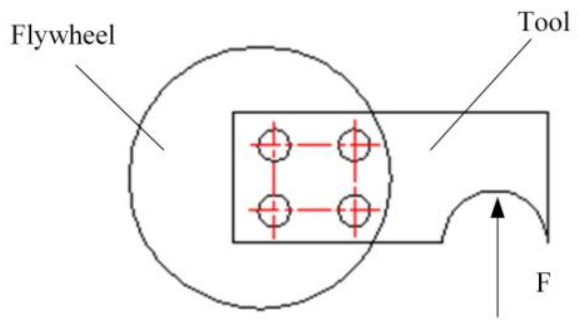

Figure 3 Tool Schematic Diagram
Because the rotary shear blanking machine need to blanking different types of metal bars, the mould uses Vshaped clamping piece to clamping, so that, it is convenient to replace the corresponding mold in the production of different types of bars. Adopts the form of radial convex platform fit into groove to realize axial fixation of the mould. A slot in the V-shaped block is open in order to connected to the radial of the hydraulic cylinder, bolted is used in axial connection to achieve the hydraulic cylinder fixing with V-shaped block. Same as the shape of a machine tool guideway is designed in the front of the V-shaped blocks, and cooperate with base hole corresponding to ensure $\mathrm{V}$-shaped block only move along the radial direction, it can realize accurate clamping[9]. The mould can increase the contact area when the metal bar is blanking and reduce the deformation of the shear section on the metal bar. The convex platform in the center of the die is to prevent the axial movement of the mould. The shear mold structure is shown in Fig. 4.

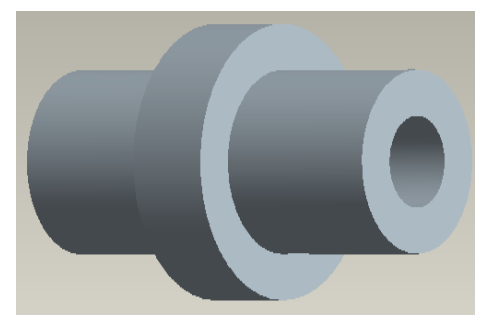

Figure 4 Blanking Die

\subsection{The shearing blanking system and feeding mechanism}

In the initial state, the bars are arranged on a table with slope, and then two hydraulic cylinders rise at the same speed. The first bar will fall until the feeding block is intercepted and sent to the roller, during this period, the hydraulic cylinder will block the second rod falling. After the first rod is delivered, the hydraulic cylinder drops to the initial position, while the second rod is reduced to the position of the first bar. The fixed mode of hydraulic cylinder and hydraulic pump is the same as the clamping mechanism. But considering the speed of the hydraulic cylinder rising or falling too much, in the process of rising the bar avoiding pop-up deviates from the original set route, in setting speed regulating valve into the oil and out of the oil, guarantee hydraulic cylinder can quickly and stability lift and transport bar. The general assembly drawing is shown in Fig. 5.

\subsection{The fixture structure design}




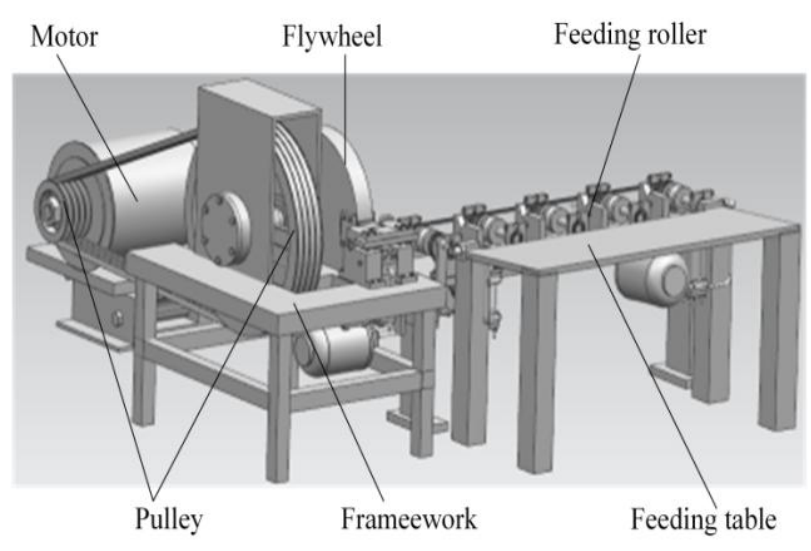

Figure 5 Shearing Blanking General Assembly

For the feeding mechanism, the whole feeding device how to accurately connect with blanking device and clamping device should to be considered. Based on this consideration, the height of the feeding device is adjusted by the friction between the bolted and similar keyway, ensure bar can accurate be sent to the clamping device. Rotary type high-speed shearing blanking system, including the feeding machine and feeding device.

\section{Structure optimization of blanking tool}

As the tool is the core part of the cutting blanking process, the strength must meet the design requirements. The finite element analysis of the cutter is shown in Fig.6.

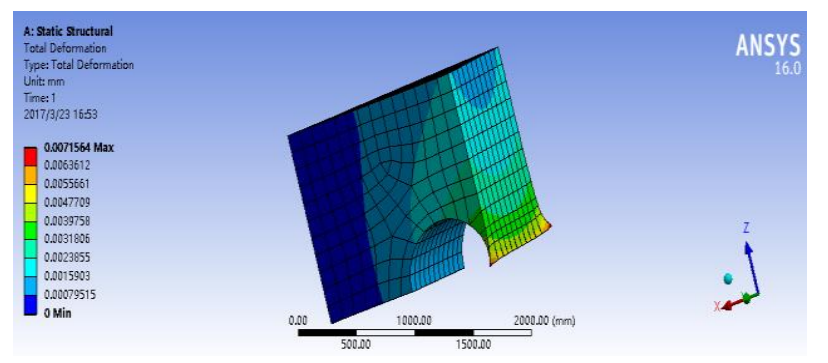

Figure 6 The Cutting Tool Stress Distribution Nephogram

From the Fig. 6, we can see that stress concentration is relatively serious in the lateral of tool opening, structure with large deformation and the tool has a larger damage. This is because the structural design of the tool is unreasonable and the shear force is too large in the blanking process. So should to increase the strength in the outside of the tooling opening, optimize the structure, reestablish the geometric model and mesh, the stress distribution cloud picture as shown in Fig.7. Stress concentration phenomenon of new structure greatly abate. The maximum stress is $0.3655 \mathrm{MPa}$ after optimizing, the stress value is reduced greatly, has been not enough to make the optimized cutting tool produces obvious deformation, and in range of the fourth intensity, therefore the optimization results can meet the strength requirement[9-10].

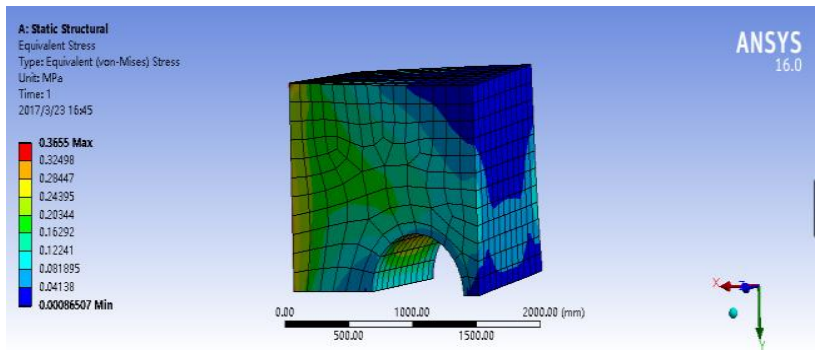

Figure 7 The Optimized Cutting Tool Stress Distribution Nephogram

\section{Experimental study}

\subsection{Test equipment}

Under $10 \mathrm{~mm}$ diameter in range of the small and medium-sized diameter bars, can be directly sheared by the rotary shear blanking machine[11-14], the picture of real products of the blanking machine as shown in Fig. 8.

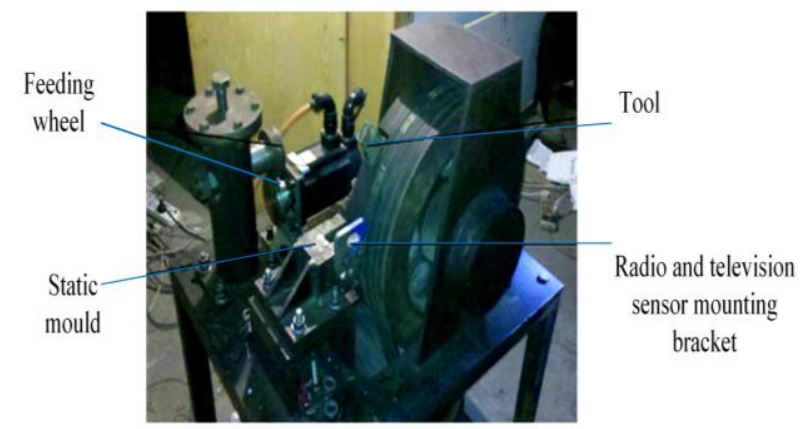

Figure 8 Pictures of the Rotary Type High-speed Shearing Blanking Machine

The feeding machine are consists of dynamic cutting edge, static mould, feeding wheel, clamping wheel, servo motor, main motor, photoelectric sensor and control system. The prefabricated circular $\mathrm{V}$ groove bar with certain geometric parameters is fixed on the holding wheel and the feeding wheel, the bar is feed by the servo motor in the signal drive of the control system, and the induction signal controlled by the photoelectric sensor signal control, each time the photoelectric sensor sensing dynamic shear blade, the servo motor feed the bar. In a set time, one of the dynamic shear blades just finishes shearing after the bar is feed.

\subsection{Experimental results and analysis}

Because the bar is in the single cantilever state in the process of shear, often caused some defects in the crosssection of bar, such as pits in one end and bumps in the other, as shown in no. 2 test bar of Fig.9. Although the pit is not very deep, affect the blanking precision. In order to solve this problem, the state of bar is to be adjust, each time a bar is feed let the servo motor feed a more certain length, let the bar be constrained on both ends, at this time, doing the high speed shearing blanking, the bar getting from the blanking as shown in no. 3 sample in 
Fig.9, and the section effect is improved. The best highest blanking speed is $150 \mathrm{piece} / \mathrm{min}$.

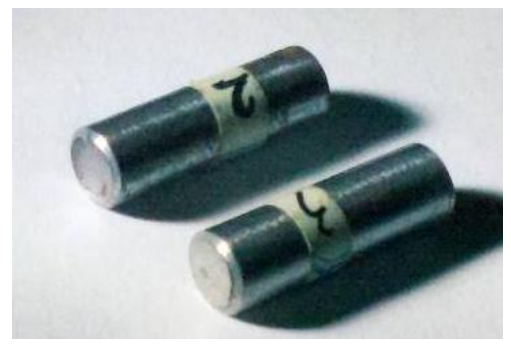

Figure 9 Ф8mm Stainless Steel Bar Stock Cutting Section

The cross-sections of the traditional shear blanking methods and rotary type high-speed shearing blanking process are shown in Fig. 10. The quality of blank section can be clearly seen from Fig.10. It can be seen that and the quality of section Fig. 10 (c) is obviously better than that of Fig.10 (a) and Fig.10 (b). The cross-section obtained from the torsional shear blanking process is shown in Fig. 10(a). The tear phenomenon is obvious on the cross-section, there are large area raised and pits, and it is poor quality. In the shear blanking methods, the torsion shear efficiency is low, punching shearing blanking process rotary type high-speed shear blanking process are more efficient. The cross-section obtained from punching shearing blanking process is shown in Fig. 10(b). The profile of the cross-section is obviously deformed, with a horseshoe shape. The cross-section obtained from rotary type high-speed shearing blanking process is shown in Fig. $10(\mathrm{c})$. The billet is no deformation at the end, with smooth section and good quality.
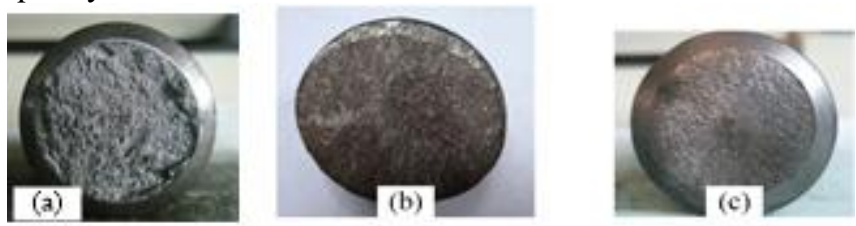

(a) Torsion Shear Process (b) Punching Shearing Blanking Process (c) Rotary type high-speed shearing Blanking Process Figure 10 Shearing Blanking Bar Section

Comparison with the punching shear process and rotary type high-speed shear blanking process, the test results of can be obtained as shown in Table 1 .

Table 1 Test Data Comparison of Three Kinds of Shear Blanking Process

\begin{tabular}{|c|c|c|c|c|c|c|c|}
\hline $\begin{array}{l}\text { Blanking } \\
\text { process }\end{array}$ & $\begin{array}{c}\text { Bar's } \\
\text { Materials }\end{array}$ & $\begin{array}{c}\text { Bar's } \\
\text { diameter }\end{array}$ & $\begin{array}{l}\text { Height } \\
\text { of bump }\end{array}$ & $\begin{array}{l}\text { Height } \\
\text { of pit }\end{array}$ & $\begin{array}{c}\text { The } \\
\text { percentage } \\
\text { of defects }\end{array}$ & $\begin{array}{l}\text { The geometry of } \\
\text { the cross- } \\
\text { section }\end{array}$ & $\begin{array}{c}\text { Blanking } \\
\text { speed }\end{array}$ \\
\hline Torsion shear & $\begin{array}{c}304 \\
\text { stainless } \\
\text { steel }\end{array}$ & $\Phi 8 \mathrm{~mm}$ & $2.25 \mathrm{~mm}$ & $3.12 \mathrm{~mm}$ & $65 \%$ & Round shape & $\begin{array}{l}15 \text { piece } \\
/ \text { min }\end{array}$ \\
\hline Impact shear & $\begin{array}{c}304 \\
\text { stainless } \\
\text { steel }\end{array}$ & $\Phi 8 \mathrm{~mm}$ & $1.15 \mathrm{~mm}$ & $2.51 \mathrm{~mm}$ & $12.5 \%$ & Oval shape & $\begin{array}{l}60 \text { piece } \\
/ \text { min }\end{array}$ \\
\hline $\begin{array}{l}\text { Rotary type } \\
\text { high-speed } \\
\text { shear }\end{array}$ & $\begin{array}{c}304 \\
\text { stainless } \\
\text { steel }\end{array}$ & $\Phi 8 \mathrm{~mm}$ & $0.35 \mathrm{~mm}$ & $0.41 \mathrm{~mm}$ & $0.5 \%$ & Round shape & $\begin{array}{c}150 \text { piece } \\
/ \mathrm{min}\end{array}$ \\
\hline
\end{tabular}

From Table 1, we can see that cross-sections of billets have the very high geometric accuracy, verticality and roughness. There are nearly no geometrical deformation and burrs, can be directly used in cold extrusion production such as gears, spline shaft and guide sleeve. However, the traditional punching shear process has the defects such as horseshoe shape, burr and so on. And the values of pits and bumps are relatively large. Especially the ratio that defects area occupies the whole section area reached $12.5 \%$, it is too more. And the whole contour of cross-section got from the punching shear blanking of has passed from the initial circle to oval. It cannot be directly used in the next production procedure. Their crosssections are shown in fig.10. Firstly defects should be treated, then it can be used for cold extrusion production such as gear, spline shaft and guide sleeve. At the same time, the rotary type high-speed shear blanking machine greatly reduces the loss of materials and also improves production efficiency.

In conclusion, the billet cross-section obtained from rotary type high-speed shearing blanking process has the very high geometric accuracy, verticality and smoothness. There are almost no defects such as geometric deformation and burrs. It can be directly used in cold extrusion production, such as gears, spline shaft and guide sleeve. However, the cross-sections got from the traditional shear blanking method have the defects such as horseshoe deformation or obvious bumps phenomenon and tear. This kind of billets with defects should go through the subsequent treatment processing. And then can be used for the cold extrusion production such as gear, spline shaft and so on. As a result, the new type of shear blanking process has greatly reduced the loss of materials and improved production efficiency. 


\section{Conclusion}

By the related test and study showed that shear rate of 6 to $7 \mathrm{~m} / \mathrm{s}$ in the high-speed rotary shear blanking process, be close to the critical brittle fracture speed of the metal, then quality of cross-section is best. Its accuracy is equivalent to the precision of the sawing machine, and retains some advantages such as die is simple and economical of the common shearing blanking.

(1) In this paper, a new type of rotary type high-speed shear precision blanking process is introduced, and the blanking machine structure and the feeding device have been designed and the experimental platform has been completed.

(2) $\Phi 8 \mathrm{~mm}$ diameter stainless steel bars were selected for experiment research, the blanking efficiency is improved and the good cross-section quality of billet is obtained. The largest convex and concave depth together with the defect proportion of cross-section are used to evaluate the quality of cross-section.

(3) The blanking technique is more suitable for medium and small diameter bar under the expected, the brittle material and plastic material for different bars should be to experiment research.

\section{References}

1. $\mathrm{Li} \mathrm{J} \mathrm{W}, \mathrm{Hu} \mathrm{C} \mathrm{L}$, Zhao Z, Li Z M .Influence of shearing speed on section quality for bar shearing $[\mathrm{J}]$. Forging \& Stamping Technology, 2016, 41(8):122126.

2. Guo B T. Current situation and developing trend of global hacksawing machines[J]. Manufacturing Technology \& Machine Tool, 2004,0(2):32-33.

3. Du S W. Bar high speed shearing machine[M]. Beijing: Metallurgical Industry Press, 2009.

4. Yu YP, Yang QH, Hu MJ. Numerical simulation on the blanking of metal tubes[J]. Mechanical Materials and Manufacturing Engineering, 2014, 455: 123-126.

5. Li Y H, Li Y T. Research and application of shearing speed of high-speed precision shearing for metal bar[J]. Hot Working Technology, 2010, 39(15): 8-11.

6. Hao B H. The analysis of blank shearing process and the methods for imporving breskan section quality $[\mathrm{J}]$. Metal Forming Technology, 2000, 18(4):44-45.

7. Zhong W, Zhao S D, Wang Z Y, Dong Y Z, Jing F. Discussion about method of improving the bar cutting surface quality through raising the hard brittle of material [J].China Metal forming Equipment \& Manufacturing Technology,2016,51(02):77-83.

8. Gao L J. A metal bar material of high speed precision shearing experimental study[D]. Taiyuan: Taiyuan University of Science and Technology, 2008.

9. Cui Y B, Wang Z, Cui Y. The notice of using V type block[J]. Science \& Technology Information, 2011,(9):27.

10. Tu Z F. ANSYS Finite element analysis of the engineering application instances tutoria[M]. Beijing: China Building Industry Press, 2010.

11. Tong Y, Zhao S D, Wang Z W. Development of a new type precision cropping machine with rotary striking $[\mathrm{J}]$. China Metal forming Equipment \& Manufacturing Technology, 2010, (06):14-17.

12. Yan H P. The designing about structure of cut equipment of high speed cut machine YAN Huiping[J]. Manofacturing Automation, 2002, 24(10): 26-32.

13. Tong Y, Zhao S D, Wang Z W. Experimental study on new precision cropping process for metal bars[J]. China Mechanical Engineering, 2010, 21(03): 359363.

14. Zhao R F, Zhao S D, Jing F, Li J X, Guo $T$. Experimental study of new precision blanking technology for thick-wall tubes[J]. Forging \& Stamping Technology, 2014, 39(8): 105-108.Here are some examples:

\section{Acknowledgement}

The authors wish to express gratitude to the Foundation of Shaanxi Province Educational Committee (Approval No. 16JK549) and Natural Science Foundation of Jiangsu Province (Approval No. BK20140407) which have supported this work. 